\title{
26 Research Square \\ Thermoplasmonic Effect Onto Toad Physiology Signals by Plasmonic Microchip Structure
}

\section{S. Akbari}

Shahid Beheshti University

S. M. Hamidi ( $\Delta$ m_hamidi@sbu.ac.ir)

Shahid Beheshti University

H. Eftekhari

Islamic Azad University

\section{A. Soheilian}

Shahid Beheshti University

\section{Research Article}

Keywords: Plasmonic chip, Surface lattice resonance, Toad, Heart signal

Posted Date: August 3rd, 2021

DOI: https://doi.org/10.21203/rs.3.rs-751790/v1

License: (9) This work is licensed under a Creative Commons Attribution 4.0 International License. Read Full License 


\section{Abstract}

Cardiovascular diseases are considered as the leading cause of death and almost $80 \%$ of deaths from this disease are developed in poor and less developed countries where early detection facilities are less available, along with overlooking the importance of screening. In other words, real-time monitoring of the physiological signals using flexible and wearable biosensors plays an important role in human life style. Thus, the present study aims to propose two dimensional flexible and wearable gold covered plasmonic samples as a physiological signal recorder, in which chips with nano array of resonant nanowire patterns performing in an integrated platform of plasmonic devices. The produced surface plasmon waves in our main chip were paired with an electric wave from the heart pulse and it use for recording and detecting the heartbeat of a toad with high accuracy. This measurement was performed in normal state and under external laser heating process to check the ability of signal recording and also thermoplasmonic effect onto the toad's heart signal. Our results show that our sensor was enough sensitive for detection while raising the body temperature of the toad and changing its heart rate as flatting $T$ and $P$ waves by thermoplasmonic effect.

\section{Introduction:}

Monitoring and distinguishing human physiological signals by portable, flexible, and wearable substrates play an important role in designing and constructing healthcare sensing devices [1-2]. Regarding the main objective of designing and fabricating flexible and wearable health monitoring devices, there are a lot of reports onto flexible batteries [3], stretchable display [4], and head of flexible main sensors [5]. These head devices are listed as main categories such as flexible piezoresistors [7], capacitors [8], carbon nanotubes [9], ZnO nanowires [10], or plasmonic nanostructures [11] which can integrate with clothes [12-13], eye glasses [14], wristwatches [15], and even skin sensors [16-17]. Enhancing signal-to-noise ratio, fast response, and remote sensing importance have motivated scientists to design and fabricate all optical sensors based on plasmonic nanostructures [18-19]. These nanostructures which can sustain collective electron oscillations at their surface to control electromagnetic field localization have been widely used for fabricating the mentioned flexible sensors based on plasmonic enhanced resonators [2021], Raman scattering [21], and photovoltaic [21] onto different substrates.

In addition, surface lattice resonance as another important phenomenon in plasmonic nanostructures has been used widely in different areas such as magneto-optical activity [22], random lasing [23], optical filters [24], and photothermal treatment by thermoplasmonic effect [25]. This efficient and periodic thermoplasmonic effect in two-dimensional (2D) plasmonic nanostructures can help us design and fabricate the head of remote sensor by the aid of 2D plasmonic structures onto flexible substrates to be used as a physiology signal recorder. Among more than seven types of flexible substrate, silk [26] and polydimethylsiloxane (PDMS) [27] are the most popular and biocompatible substrate to integrate nanosensor devices which must be prepared in the lowest thickness in a miniaturized sensor. To obtain low cost flexible sensor, the ability of another famous polyimide substrate as Kapton film is used which 
can maintain its excellent physical, electrical, and mechanical properties over a wide temperature range [28-29].

Moreover, different medical treatments and enhanced body temperature in the vicinity of heart or pulse in some diseases like heart arrhythmia can be controlled by neural stimulation and modulation based on photo thermal effects in all of optical processes [30-33]. Thus, nano-heaters can be helpful for enhancing the photo thermal effect and must be traced in heart beats. The results indicated the photo thermal effect of gold nanorods on enhancing neural activities in cells and nerves during infrared neural stimulation [34-35]. Furthermore, infrared neural stimulation of the sciatic nerve is enhanced by carbon nanoparticles [36]. In addition, a two-dimensional structure based on gold nanorods can help us benefit from infrared neural stimulation for regulating membrane depolarization [37].

Innovations in miniaturized sensor technologies have made it possible to record electric impulses from heart in the absence of conventional electrocardiography (ECG) machines. Many of such technologies are wearable and can record cardiac impulses for long periods of time, which can lead to an increase in the utility of this technique in out-of-hospital settings such as households, endurance training, sports training, and public places. Further. it is possible to immediately transmit the obtained waveforms for expert interpretation, along with the already available computerized reports [38]. These sensors should have flexible and wearable properties, and should be small enough in size, which can be used for monitoring rhythms and waveforms over weeks or months. Thus, as another main part of remote sensors, all optical miniaturized and low size branches must be applied in the system. For this purpose, we use the pickup head of conventional DVD-ROM including a semiconductor laser diode, a lens for focusing on the laser beam, and photodiodes for detecting the light reflected from the disc surface.

The above-mentioned head and detection part present a simple plasmonic, low cost, flexible, and handheld sensor for physiological signal sensing and trace of photo thermal effect onto the heart beat instead of common ECG systems.

\section{li. Materials And Method:}

As the main head of signal recorder, 2D plasmonic nano structure has been fabricated by nano imprint lithography onto the Kapton substrate as follows. First, a CCD was extracted and separated from a camera. Then, a layer of the Kapton tape was placed onto the CCD by applying pressure. In the next stage, the sample was placed on the heater at $75^{\circ} \mathrm{C}$ for 30 minutes. The sample was maintained under pressure at the room temperature for one week to stabilize the two-dimensional pattern onto the Kapton tape. Then, the Kapton tape was carefully removed from the CCD after one week, and a 2D flexible structure with very low thickness was achieved. Finally, a thin layer of gold with a thickness of $35 \mathrm{~nm}$ was deposited on the 2D Kapton substrate. Accordingly, a 2D flexible and very thin plasmonic crystal consisting of Kapton-Au was produced. 
After characterizing surface by scanning electron microscopy (SEM) and recording surface lattice resonance (SLR) of the sample in the visible region, microchip pickup head of conventional DVD-ROM was used (Fig. 1a). The head consisted of a semiconductor laser diode, $680 \mathrm{~nm}$, with $5 \mathrm{~mW}$ power, a lens for focusing the laser beam, and photodiodes for detecting the light reflected from the 2D plasmonic surfacewhich put onto the fixed toad, and three immature Eurasian green toads (weight $50-70$ grams) on the optical set-up (Fig. 1b). Finally, the reflected signals from toads were collected by digital oscilloscope.

As shown, the sample at SLR wavelength has more sensitivity and can distinguish and follow the input signal more in PQR region of the hart signal instead of other areas. There is the dependency of the reflections under $\mathrm{p}$ - and s-polarized incident light on the frequency and current from two-dimensional plasmonic sample, which comes from the charge redistribution at the interface influencing the plasmonic resonance, especially SLR of the main head sample.

During the second step, continuous $532 \mathrm{~nm}$ laser irradiation fixed at the distance of $5 \mathrm{~cm}$ from the top of the toad onto main head was used to trace thermoplsmonic effect onto heart signals. First, the toad heart area was illuminated just by laser, and then plasmonic substrate was added to the heart area to determine the different effects of Au nano-rods heating and laser radiation. Regarding the required time for increasing the temperature, the heart area was irradiated for more than 5 minutes (Fig. 1b).

As shown, the microchip was placed on the top of the toad heart after fixing the green laser. The arrangement should be in such a way that the green laser can hit only the two-dimensional sample on the toad heart without damaging the microchip. In this experiment, the temperature was measured by a thermocouple (TM-916, LUTRON electronic enterprise co, Taiwan). All of the tests were performed under the same conditions at a laboratory temperature of about $23^{\circ} \mathrm{C}$. After time and local heating, the microchip was turned on and the toad heart signal was measured after heating. Thus, the use of anesthetics such as ether or chloroform interferes with the normal heart rhythm and heart rate of the toad [39]. Thus, no anesthetic was used in this experiment.

When we shine a $532 \mathrm{~nm}$ pump laser at three different powers $(10,60$, and $100 \mathrm{MW})$ over time, a layer of gold particles heats up on the sample, and transfers heat to the toad skin and the underlying tissue. To confirm the assumption, the sample was simulated in a commercial finite element method (FEM) solver, COMSOL Multiphysics 5.2 to approve the warming of the toad skin, which transfers to the tissue.

To simulate surface lattice resonance (SLR) of the sample, Wave Optics Module and the Electromagnetic Waves, and Frequency Domain interface were used. In addition, a plane TE-polarized electromagnetic wave propagation was modeled in a single unit cell of a gold layer on a Kapton substrate. Based on the electron microscope images, the thickness of the Kapton and gold layer were considered as 6 micrometers and $35 \mathrm{~nm}$, respectively.

\section{Sample guideline:}

All methods were carried out in accordance with relevant guidelines. 
All experimental procedures were performed in accordance with guidelines and regulations approved by a regional Institutional Animal Care and Use Ethics Committee of the "Ethical committee of Vice president of research of Shahid Beheshti university/IR.SBU.REC.1405".

\section{lii. Results And Discussion:}

Figure 2(a) displayed the SEM of the fabricated sample indicating nanowires in the corner of each unit cell by the size of 650 in $100 \mathrm{~nm}$. In addition, real picture of the main flexible 2D plasmonic chip is shown in Fig. 2(b). Due to lattice resonance of these wires, surface lattice resonance (SLR) is observed in the middle of visible region (Fig. 2c). Thus, we have good answer onto the detector from this sample in the visible region which must be useful as the sensor of flexible and ultra-sensitive physiological signal to heart pulse diagnostics. Due to the warming of the tissue, a sudden increase occurred in heart rate, and our sensor could detect this signal change well.

As explained above, heart pulses were recorded by the aid of our 2D plasmonic chip and microchip array, as shown in Fig. 2(d). To get more sense about the heart signal, in the top row of this figure, we used the normal heart rhythm of this kind of toad picked up from [40-41]. In the middle row, the recorded pulses by $2 \mathrm{D}$ plasmonic chip were considered and pulses were recorded after green laser pumping onto $2 \mathrm{D}$ plasmonic chip and thus thermoplasmonic effect in the last ones (bottom row). Heart rhythm phases depend directly on the activity of the heart muscle and are affected by ionic changes within the heart cells. Due to the heating of gold Nanorods on a 2D plasmonic chip, the results of toad heart stimulation indicated significant changes in the activity of toad heart. Although no change was observed in heart rate when no thermal stimulation was performed and the toad was at normal temperature conditions, the effect of the green laser on the 2D sample and the heating of the gold nanorods had a significant effect on the heart rate. In addition, the ability of our sensor to detect and record the transmission of cardiac signals and their reproducibility is confirmed when the green laser was off.

In fact, when the plasmonic nanostructure is fixed on the toad heart and the immobile toad is placed at a suitable distance from the microchip, the toad heart signal recording is performed well. Thus, the signal recorded by our sensor matches very well with the heartbeat signal of an adult toad.

As shown in second row in Fig. 2 (d), no change in the amplitude of the heart rhythm phase was seen. However, the local temperature increase in the toad heart area due to the thermoplasmonic effect of gold nanorods onto the main 2D plasmonic grating, it affects the $T$ and $P$ waves amplitude. Compared to the toad heart rate rhythm before heat stimulation by green laser, the $T$ and $P$-waves amplitude is significantly decreased. The decrease and flattening effect in these waves amplitude is due to some biological effects on the toad's heart due to increase in temperature and heating.

It has been shown that increasing the temperature is effective in reducing the Action Potential time [42] and thus decrease in $\mathrm{T}$ and $\mathrm{P}$ waves amplitude in our experiment onto toad's heart which is probably due to the increase in temperature that was transmitted to the underlying tissue through the toad's skin and affected the heart rate. Another reason is the increase in the concentration of calcium ions inside the cells 
[43]. It was shown that temperature increase by $I R$ laser led to an increase in intracellular free $\mathrm{Ca}^{2+}$ concentration in ventricular cardio myocytes and cortical neurons [44-45]. In summary, our results show the photothermal effects that gold particles have on heart cells and thus on heart activity, and our sensor was able to accurately detect changes in the heart signal. In fact, our sensor had the ability to record the toad's heartbeat signal both in the normal state and in the state of change in heart activity.

To get more sense about this thermoplasmonic effect, the main 2D plasmonic chip was simulated. In fact, the SLR of the sample was obtained as electric field distribution and reflectance spectra of the sample (Fig. 3a-b), which confirms that the plasmonic structure exhibits two resonant behaviors at 660 and $780 \mathrm{~nm}$ wavelengths by sweeping the wavelength.

Then, the incident heat flux was modelled from the laser by green pumped laser, which distributed heat source spatially on the sample. Thus, the transient thermal response of the sample was modeled with Heat Transfer in Solids Module in COMSOL.

To this aim, the sample was irradiated by a Gaussian laser beam and heated for 0.4 minute while the laser power was held constant at $10 \mathrm{~mW}$. Figure 3 (c) displays the probe plots of the average temperature distribution across the sample for different distances of the laser focal point $(h)$. Figure 3(d) shows the temperature distribution plot across the sample for different sweeping time.

\section{Iv. Conclusions:}

In this study, new generation of wearable, small, flexible, and light heart rate recorders was proposed based on two dimensional plasmonic nanostructures as the main chip onto Kapton substrate. To reach the portable recorder, miniaturized optical setup was used near the heart surface of the toad. Based on the results, very good agreement was observed between input and output signal of heart beats in the toad and ability to distinguish between different signals. Finally, thermopalsmonic effect due to gold nanorods onto our main chip under green laser pump could affect the T and P shape of the heart signal, which was confirmed by the aid of the simulation part.

\section{Declarations:}

\section{Author contributions:}

S Akbari did the optical measurement, analyzed the results and wrote the main text of the manuscript. A. Soheilian simulate the thermoplasmonic part, $\mathrm{H}$ Eftekhari control microchip alignment and S.M.Hamidi. supervise the measurement part and writing the results of the study. All authors read and approved the final manuscript.

\section{Competing interests:}

The authors declare no competing interests. 


\section{References:}

1. A. Bansal and R. Joshi, "Portable out-of-hospital electrocardiography"A review of current technologies, J. Arrhythmia, vol. 34, no. 2, 129-138,(2018).

2. S.Majumder, T.Mondal, M.J.Deen," Wearable sensors for remote health monitoring". Sensors, 17, 130, (2017).

3. Sh.H.Liu, J.J.Wang Ch.H. Su, D.Ch .Cheng, "Improvement of left ventricular ejection time measurement $\mathrm{n}$ the impedance cardiography combined with the reflection photoplethysmography". Sensors, 18, 3036, (2018).

4. Y.Liu, Q.Liu, SH.Chen, F.Cheng, H.Wang, W.Peng, "Surface Plasmon Resonance Biosensor Based on Smart Phone Platforms". Sci Rep 5, 12864, (2015).

5. C.C.Hsu, B.S.Lin, K.Y.He,B, "Design of a Wearable 12-Lead Noncontact Electrocardiogram Monitoring System" Sensors, vol. 19, no. 7, p. 1509,(2019).

6. V.A.Convertino, S.G.Schauer, E.K.Weitzel, S.Cardin, M.E.Stackle, M.J.Talley, M.N.Sawka, O.T. Inan, "Wearable Sensors Incorporating Compensatory Reserve Measurement for Advancing Physiological Monitoring in Critically Injured Trauma Patients".Sensors (Basel). 10;20(22):6413, (2020).

7. D. H. Kim, N. Lu, R. Ma, Y. S. Kim, R. H. Kim, S. Wang, J. Wu, S. M Won, H. Tao, A. Islam, K. J. Yu, T. I. Kim, R. Chowdhury, M. Ying, L. Xu, M. Li, H. J. Chung, H. Keum, M. McCormick, P. Liu, Y. W. Zhang, F. G. Omenetto, Y. Huang, T. Coleman, J. A. Rogers, "Epidermal Electronics" Science, 333, 838,( 2011).

8. W. Gao, S. Emaminejad, H. Y. Y. Nyein, S. Challa, K. Chen, A. Peck, H. M. Fahad, H. Ota, H. Shiraki, D. Kiriya, and D. H. Lien, "Fully integrated wearable sensor arrays for multiplexed in situ perspiration analysis" Nature 529, 509-514, (2016).

9. G. Schwartz, B. C. K. Tee, J. Mei, A. L. Appleton, D. H. Kim, H. Wang, and Z. Bao,"Flexible polymer transistors with high pressure sensitivity for application in electronic skin and health monitoring", Nature communications 4 ,1859-1866, (2013).

10. F. Sohrabi, M. Kiaei, T. Mahinroosta, and S. M. Hamidi, "Phase-Sensitive Pulse Sensor Using 2-D Active Plasmonics on Conformal Substrates", IEEE Transactions on Electron Devices 67,4379-4384, (2020).

11. H.Wang, D.W.Brandl, P.Nordlander, N.J.Halas,"Plasmonic Nanostructures": Artificial Molecules,Accounts of Chemical Research ,40 (1), 53-62, (2007).

12. L.H.Dai, H.Z.Zhao, X.Zhao, Y.J Zhou,"Flexible and printed microwave plasmonic sensor for noninvasive measurement" IEEE Access, vol. 8, pp. 163238-163243, (2020).

13. J. Zhong, Y. Zhang, Q. Zhong, Q. Hu, B. Hu, Z. L. Wang, J. Zhou," Fiber-Based Generator for Wearable Electronics and Mobile Medication", ACS Nano, 8, 6273, (2014).

14. S.Feng, R.Caire, B.Cortazar, M.Turan, A.Wong, A.Ozcan, "Immunochromatographic Diagnostic Test Analysis Using Google Glass",ACS Nano, 8, 3069,(2014).

15. D.J.Wile, R.Ranawaya, Z.H.T.Kiss," Smart watch accelerometry for analysis and diagnosis of tremor",Journal of Neuroscience Methods,Volume 230, Pages 1-4, (2014). 
16. D. H. Kim, N. Lu, R. Ma, Y. S. Kim, R. H. Kim, S. Wang, J. Wu, S. M. Won, H. Tao, A. Islam, K. J. Yu, T. I. Kim, R. Chowdhury, M. Ying, L. Xu, M. Li, H. J. Chung, H. Keum, M. McCormick, P. Liu, Y. W. Zhang, F. G. Omenetto, Y. Huang, T. Colema,J.A.Rogers,"Epidermal Electronics"Science, 333, 838,( 2011).

17. X. Wang, Y. Gu, Z. Xiong, Z. Cui, T. Zhang," Silk-Molded Flexible, Ultrasensitive, and Highly Stable Electronic Skin for Monitoring Human Physiological Signals", Adv. Mater, 26, 1336, (2014).

18. M. M. Tharwat, H. AlSharif, H. Alshabani, E. Qadi , M. Sultan, "Design of an optical sensor based on plasmonic nanostructures", Metamaterials X, pp. 98830, (2016).

19. B. Špačková, P. Wrobel, M. Bocková and J. Homola, "Optical Biosensors Based on Plasmonic Nanostructures": A Review, in Proceedings of the IEEE, vol. 104, no. 12, pp. 2380-2408, Dec. (2016).

20. M. Kahraman,P. Daggumati,O.Kurtulus, E. Seker, S.Wachsmann-Hogiu," Fabrication and Characterization of Flexible and Tunable Plasmonic Nanostructures", Sci. Rep, 3, 3396, (2013).

21. L. Britnell, R. M. Ribeiro, A. Eckmann, R.Jalil, B. D.Belle, A. Mishchenko,Y. J.Kim, R.V.Gorbachev, T.Georgiou, S.V. Morozov, A. N.Grigorenko, A. K.Geim, C.Casiraghi, A. H. Castro Neto, K.S.Novoselov," Strong Light-Matter Interactions in Heterostructures of Atomically Thin Films",Science, 340, 1311, (2013).

22. H. M.barak, R.T.Ghahrizjani, S.M Hamidi, E Mohajerani, Y.Zaatar, "Reversible and tunable photochemical switch based on plasmonic structure", Scientific Reports 10 (1), 1-7,(2020).

23. S. F. Haddawi, H. R. Humud, S. A. Monfared, S. M. Hamidi," Two-dimensional plasmonic multilayer as an efficient tool for low power random lasing applications", Waves in Random and Complex Media, 1-10, (2021).

24. N. Roostaei, H. Mbarak, S. A. Monfared, S. M. Hamidi, "Plasmonic wideband and tunable absorber based on semi etalon nano structure in the visible region", Physica Scripta 96 (3), 035805, (2021).

25. N. S. Shnan, N. Roostaei, S. M. Hamidi, "Tunable and reversible thermo-plasmonic hot spot imaging for temperature confinement", Journal of Theoretical and Applied Physics 14 (4), 367-376, (2020).

26. F.G.Omenetto, D.L.Kaplan, "A new route for silk" ,Nature Photon 2, 641-643 (2008).

27. A.Mata, A.J.Fleischman, S.Roy."Characterization of Polydimethylsiloxane (PDMS) Properties for Biomedical Micro/Nanosystems", Biomed Microdevices 7, 281-293 (2005).

28. E.T.Arakawa, M.W.Williams, J.C.Ashley, L.R.Painter,"The optical properties of Kapton: Measurement and applications",Journal of Applied Physics 52, 3579, (1981).

29. P.D.Cunningham, N. N.Valdes, F.A.Vallejo, L.M.Hayden, B.Polishak, X. H.Zhou, J.Luo, A.K.Jen, J.C.Williams, R.J.Twieg, "Broadband terahertz characterization of the refractive index and absorption of some important polymeric and organic electro-optic materials" Journal of Applied Physics 109, 043505, (2011).

30. A.D.Jose, F.Stitt, D. Collison, "The effects of exercise and changes in body temperature on the intrinsic heart rate in man", Am Heart J, 79(4): 488-98, (1970).

31. N.Kleitman, A.Ramsaroop, "Periodicity in body temperature and heart rate", Endocrinology, 43(1):120,(1948). 
32. D.Dalecki, B.B.Keller, C.H.Raeman, E.L.Carstensen, "Effects of pulsed ultrasound on the frog heart: I. Thresholds for changes in cardiac rhythm and aortic pressure",Ultrasound in Medicine \& Biology, Volume 19, Issue 5, (1993).

33. H.Gong, Y.Yang, X.Chen, D.Zhao, X.Chen, Y.Chen, M.Yan, Q.Li, M.Qiu," Gold nanoparticle transfer through photothermal effects in a metamaterial absorber by nanosecond laser", Sci Rep 4, 6080 (2014).

34. G.Cosoli,S.Spinsante, F.Scardulla, L. D'Acquisto, L.Scalise, "Wireless ECG and cardiac monitoring systems: State of the art, available commercial devices and useful electronic components",Measurement,Volume177, 109243,ISSN 0263-2241,) 2021(.

35. L.Susu, A.Campu, A.M.Craciun, A.Vulpoi, S.Astilean M.Focsan, "Designing Efficient Low-CostPaperBased Sensing Plasmonic Nanoplatforms",Sensors, 18, 3035, (2018).

36. M.Q.Wang, N. Xia, X.Y.Wu, N.Hu, X.L.Zheng, W.S. Hou, "980nm infrared neural stimulation of sciatic nerve enhanced by carbon nanoparticles," 2017 8th International IEEE/EMBS Conference on Neural Engineering (NER), pp. 215-218, (2017).

37. S.Saeidifard, F.Sohrabi, M.H.Ghazimoradi, S.M.Hamidi, Sh.Farivar, M.AAnsari."Two-Dimensional Plasmonic Biosensing Platform: Cellular Activity Detection under Laser Stimulation", Journal of applied physics, 126 (10), 104701, (2019).

38. W.Gao, S.Emaminejad, H.Y.Y.Nyein, S.Challa, K.Chen, A. Peck, H. M. Fahad, H.Ota, H.Shiraki, D.Kiriya, D.H. Lien, G.A.Brooks, R.W.Davis, A.Javey, "Fully integrated wearable sensor arrays for multiplexed in situ perspiration analysis",Nature 529, 509-514, (2016).

39. Y.Cakir, S.M.Strauch, "Tricaine (MS-222) is a safe anesthetic compound compared to benzocaine and pentobarbital to induce anesthesia in leopard frogs (Rana pipiens)", Pharmacological reports: PR 57, 467-474,(2005).

40. R.W.Fasciano II, L.Tung "Factors governing mechanical stimulation in frog hearts". Am J Physiol; 277: H2311-H2320, (1999).

41. O.Rougier, G.Vassort, Y.M.Gargouil, E.Coraboeuf , "Existence and role of a slow inward current during the frog atrial action potential", Pflugers Arch. 308, 91-110, (1969).

42. F.Kong, R. Jiao, K. Liu, X. Han, C.Sun, "Continuous infrared laser irradiation decreased membrane capacitance of neuron cell" in (SPIE-Intl Soc Optical Eng), 11190, p. 70,( 2019).

43. X.R.Fan, J.H.Ma, W.Wan, P. H.Zhang, C.Wang, L.Wu, "Increased intracellular calcium concentration causes electrical turbulence in guinea pig ventricular myocytes" Sci. China Life Sci. 54(3), 240-247, (2011).

44. G. M. Dittami, S. M. Rajguru, R. A. Lasher, R. W. Hitchcock, R. D. Rabbitt, "Intracellular calcium transients evoked by pulsed infrared radiation in neonatal cardiomyocytes" J. Physiol. 589(6), 12951306,(2011).

45. A. Kaszas, G. Szalay, A. Slézia, A. Bojdán, I. Vanzetta, B. Hangya, B. Rózsa, R. O'Connor, and D. Moreau, "Two-photon GCaMP6f imaging of infrared neural stimulation evoked calcium signals in mouse cortical neurons in vivo" Sci. Rep. 11(1), 1-18, (2021). 


\section{Figures}

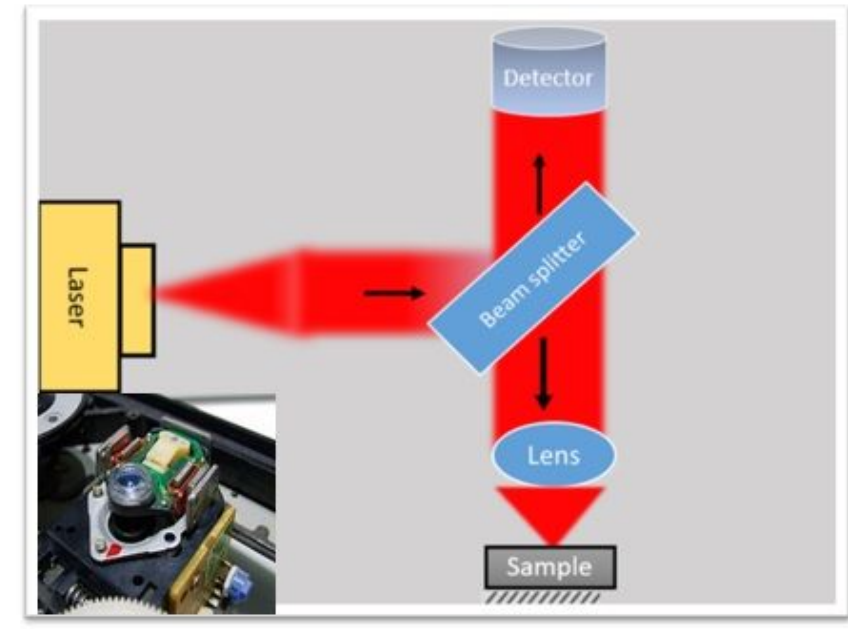

(a)

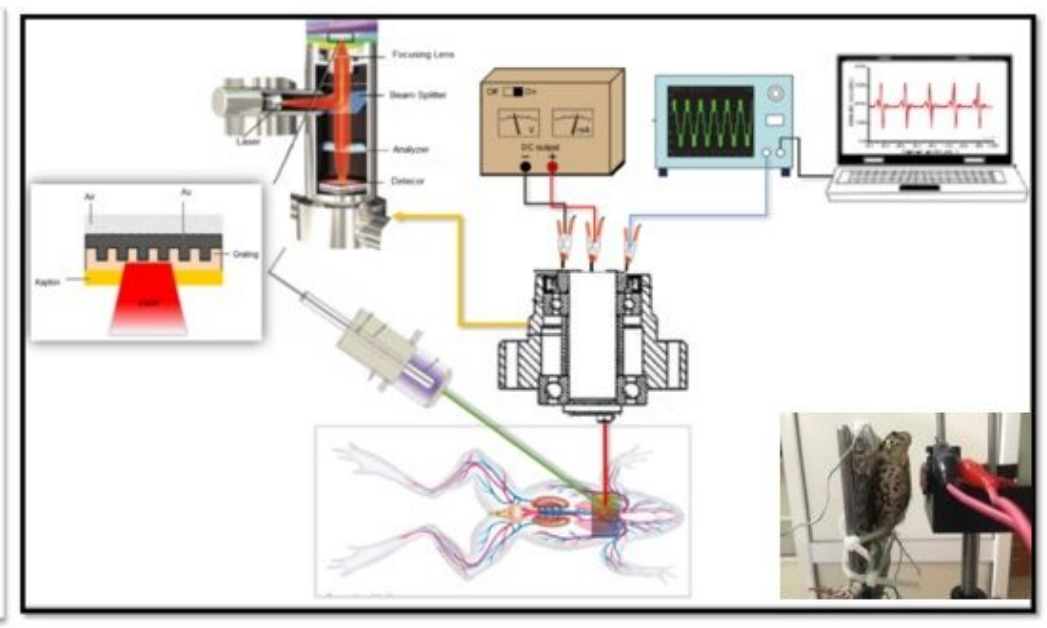

(b)

Figure 1

(a) optical setup of microchip and (b) main experimental setup which use for signal recording with and without green laser pumping. 


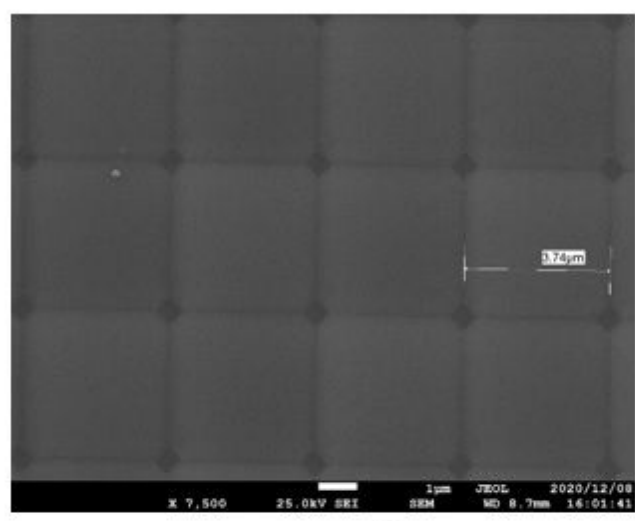

(a)

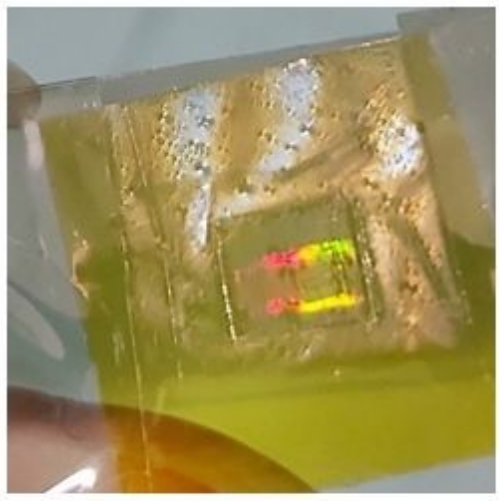

(b)

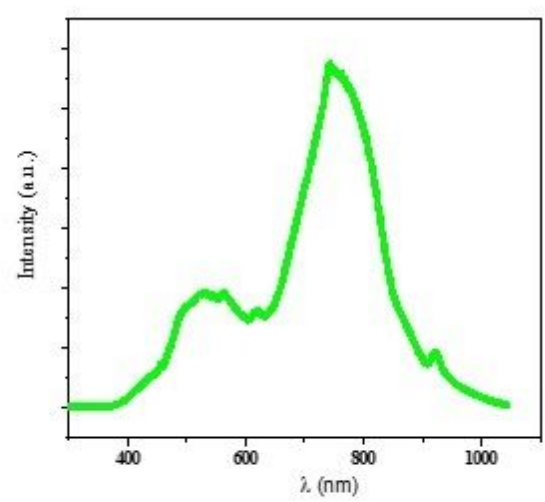

(c)

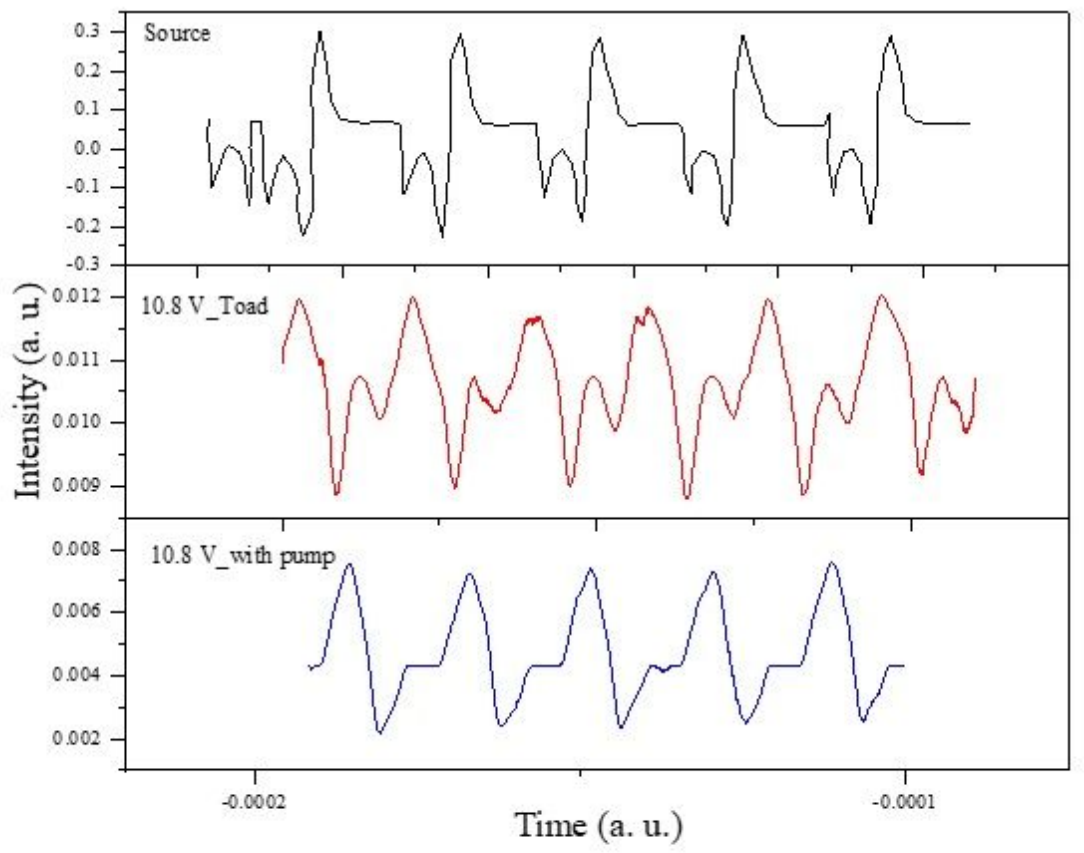

(d)

\section{Figure 2}

(a) SEM image of 2D plasmonic chip, (b) Real picture of 2D flexible plasmonic chip onto Kapton and (c) SLR response of the main 2D plasmonic chip, and (d) Heart signal recorded from toad as reference signal [46], main signal and pumped ones. 

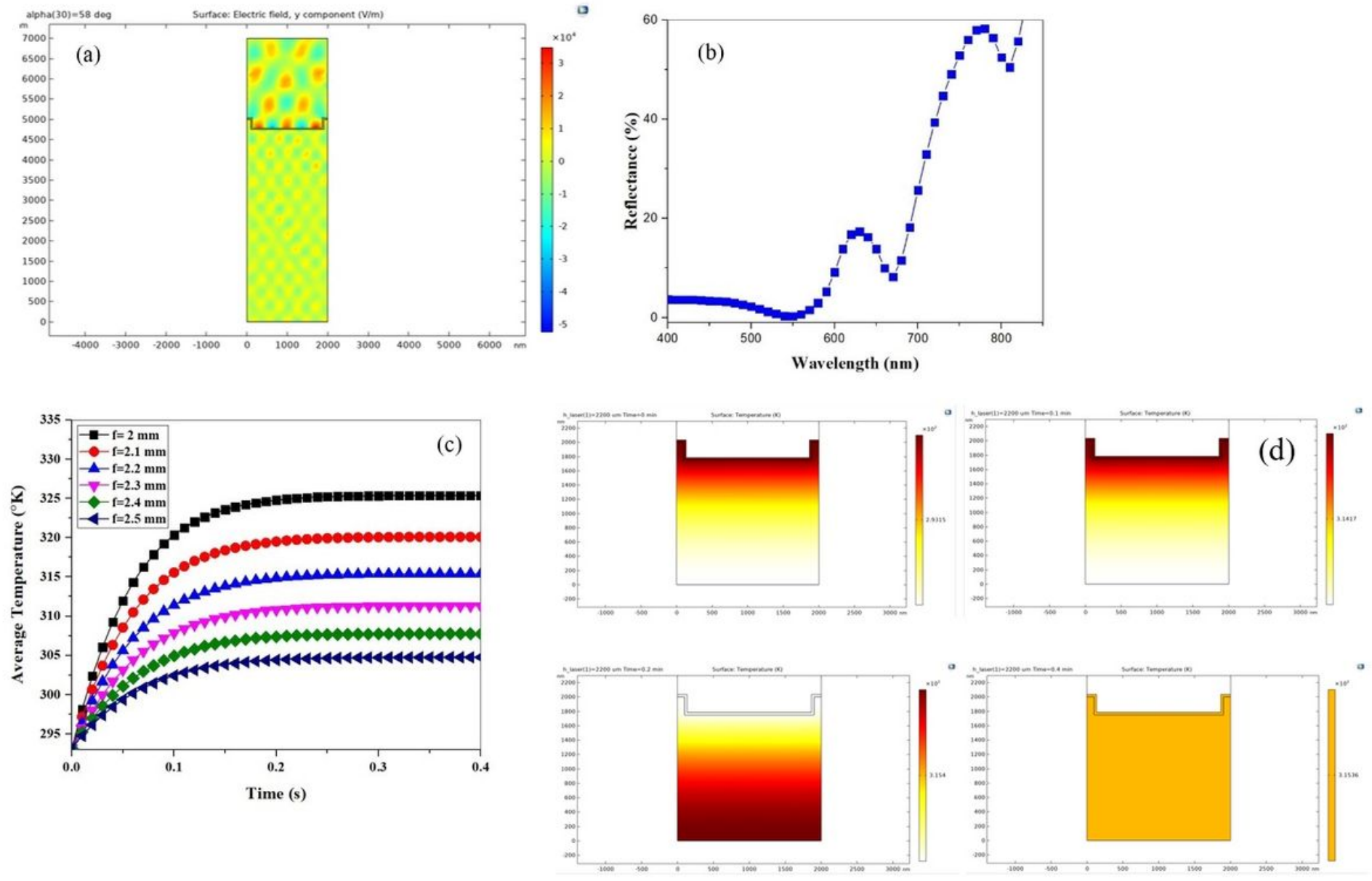

Figure 3

(a) Plasmonic SLR for propagation of plane wave at angle of $45^{\circ}$ and wavelength of $680 \mathrm{~nm}$, (b) Transmittance, reflectance and absorption of the first order diffraction of plane waves for the single unit cell. (c) The average temperatures distribution across the sample for different distance of the laser focal point (h) and (d) The temperature distribution across the sample is plotted for different sweeping time. 